\section{Comportamiento del consumidor y su proceso de decisión de compra. El nuevo camino del consumidor}

\author{
Consumer behavior and its process in making their buying \\ decisions. The new consumer's path
}

\begin{abstract}
RESUMEN
Nos encontramos frente a un mercado muy dinámico, al cual se le agrega la transformación digital y el efecto de la pandemia por el COVID-19, nuestro consumidor es mucho más informado y exigente, cuyas decisiones de compra van a depender no sólo de él sino de sus interacciones con otros consumidores y las diferentes formas de interactuar usando los medios digitales. Por ello, el principal objetivo del artículo es realizar una revisión del cambio que viene teniendo el comportamiento del consumidor y su proceso de decisión de compra y cómo las empresas deben tomar en cuenta el nuevo camino del consumidor y así desarrollar sus estrategias de marketing que estimulen la decisión de compra logrando una experiencia con el consumidor capaz de crear vínculos emocionales profundos y que concluya con la elección y la fidelización de la marca; por ello se ha elaborado un cuadro matriz que refleja la evolución de este consumidor, así su probabilidad de que se vuelva a repetir una compra asegura la permanencia de la empresa en el mercado.
\end{abstract}

Palabras Clave: Comportamiento del consumidor; consumidor; decisión de compra; marca; estrategia.

\begin{abstract}
We are facing a very dynamic market, to which is added the digital transformation and the effect of the COVID-19 pandemic, our consumer is much more informed and demanding, whose buying decision will depend not only on him but on their interactions with other consumers and the different ways of interacting using digital media. Therefore, the main objective of the article is to review the change that consumer behavior and its buying decision process have been having and how companies must take into account the new path of the consumer and thus develop their marketing strategies that stimulate the buying decision by achieving an experience with the consumer capable of creating deep emotional bonds and that concludes with the choice and loyalty of the brand; for this reason a matrix
\end{abstract}

Alicia Elizabeth Gonzales Sulla agonzales@sagafalabella.com.pe ORCID: https://orcid.org/00000002-6889-9409

Saga Falabella S.A. Lima, Perú

Presentado: 14/03/2021 - Aceptado: 31/08/2021 - Publicado: 23/12/2021

(C) Los autores. Este artículo es publicado por la revista Gestión en el Tercer Milenio de la Facultad de Ciencias Administrativas Universidad Nacional Mayor de San Marcos. Este es un artículo de acceso abierto, distribuido bajo los términos de la licencia Creative Commons Atribución 4.0 Internacional (CC BY 4.0) [https://creativecommons.org/licenses/by/4.0/deed.es] que permite el uso, distribución y reproducción en cualquier medio, siempre que la obra original sea debidamente citada de su fuente original. 
table has been prepared that reflects the evolution of this consumer, so the probability of doing a buying it again will reassure the permanence of the company in the market.

Keywords: Consumer behavior; consumer; buying decision; brand; strategy.

\section{INTRODUCCIÓN}

Hoy las empresas y los consumidores están enfrentados a nuevos desafíos. La transformación digital, el cambio tecnológico y los efectos de la pandemia del COVID-19 nos pone frente a un consumidor exigente, mucho más informado, un consumidor que compra de manera distinta, a todo ello le sumamos a la competencia en productos o servicios que en el día a día no sólo muestran estrategias de marketing en segmentación, posicionamiento y diferenciación sino que va más allá, en búsqueda de captar a este cliente, cautivarlo y haciéndolo más leal a su marca o negocio. Es por ello que, debemos revisar el modelo de negocio que estamos trabajando, bajo conceptos renovados y actualizados. El presente artículo, desarrolla conceptos y definiciones de comportamiento del consumidor y proceso de decisión de compra del consumidor, conceptos que están en el foco de las empresas o negocios, sin embargo, por la rapidez o inmediatez en la cual se mueve el mercado, las empresas no están tomado la importancia del caso, por ello, es el momento de ponerle mayor énfasis a su análisis y visualizar cómo es el nuevo camino del consumidor.

Tengamos en cuenta que el marketing está siendo más holístico en sus conceptos y más renovado en este proceso de cambios, por ello, el comportamiento del consumidor ha tomado prestado concepto de otras disciplinas científicas "como la psicología (el estudio del individuo), la sociología (el estudio de los grupos), la psicología social (el estudio del individuo en la sociedad) y la economía, para sentar las bases de la nueva disciplina de marketing" (Shiffman y Kanuk, 2005, p. 19). De esta manera se considera al consumidor como un todo, es decir, individuo, grupo, forma y su economía.

En el presente artículo revisaremos los conceptos de comportamiento del consumidor, y sus diferentes teorías y factores que la afectan, desde el punto de vista del marketing tradicional. Luego, de haber revisado las influencias que afectan al consumidor se examinará el comportamiento de la decisión de compra del consumidor que varía según el producto o servicio, por ejemplo, es muy distinto la compra de productos de consumo masivo a la de electrodomésticos, automóviles u otros. Al mismo tiempo, ingresaremos al mundo del proceso de decisión de compra del consumidor tradicional y al proceso de decisión de compra más digitalizado. Para ello, se toma los aportes de diferentes autores, dando paso a la elaboración de un cuadro matriz en la cual se visualiza y refleja cómo ha ido evolucionando el proceso de decisión de compra. De tal forma que, no se deja de lado las variables de marketing (producto, precio, plaza y promoción) sino que se le suma las 5 As (aware/conocimiento, appeal/atraer, ask/pedir, act/actuar y advocate/recomendar) conceptos actualizados en el proceso de decisión de compra del consumidor y los beneficios que acarrea como la captación de este consumidor y mantenerlo leal a la marca haciéndole vivir una experiencia única.

Finalmente, con los conceptos desarrollados se podrá mostrar el nuevo camino del consumidor de hoy y aprovechar las oportunidades del mercado, por ello es vital el uso de nuevas herramientas para el análisis profundo del consumidor, de manera que se maximice sus ventas y minimice sus gastos de campaña publicitarias y que los esfuerzos desplegados brinden los resultados esperados para la empresa y para el cliente.

\section{OBJETIVO DEL ARTÍ́CULO}

El presente artículo tiene por finalidad realizar una revisión de la evolución que está teniendo el comportamiento del consumidor en su proceso de decisión de compra, brindando al lector una información no sólo teórica sino actualizada de un mercado físico y digital. El conocer el comportamiento del consumidor y su proceso de compra permite comprender: qué compra, por qué lo compra, cuándo lo compra, dónde lo compra, con qué frecuencia lo compra y usa, cómo y dónde quiere recibirlo; 
todas estas interrogantes permiten beneficios para el consumidor y la empresa; para el consumidor porque obtendrá el producto a un precio correcto en un lugar requerido con una experiencia de compra y a la empresa que le permite incrementar las ventas y la fidelización de su marca.

Se explicará, en principio el comportamiento del consumidor basado en teorías de comportamiento de conducta, luego se revisará los factores que afectan este comportamiento y posteriormente se analizará el comportamiento de la decisión del proceso de compra del consumidor y cómo ha ido evolucionando en estos años. Al respecto Kotler et al. (2017) nos muestra justamente esa evolución para un mercado físico, digital o ambos. Por ello, la importancia de tener claridad de cómo es este nuevo camino del consumidor; se ha elaborado una matriz de esta evolución del proceso de decisión de compra físico y digital (ver Figura 1), de manera que sea aprovechada por las empresas en sus estrategias de producto, marketing, comunicación, logística entre otras y sobre todo realizar los cambios que sean necesarios cuando este camino del consumidor no está llegando a concretar las ventas esperadas. Conocer a nuestros consumidores hoy en día no es tarea fácil ya que al interactuar con otros consumidores o puntos de contacto influye en el cierre de ventas.

Finalmente, se ha desarrollado el nuevo camino del consumidor con herramientas actualizadas que permita dar un enfoque distinto al acostumbrado, de manera que contribuya a rastrear al cliente, deleitarlo y convertirlos en defensores leales de la marca.

\section{ARGUMENTACIÓN}

El comportamiento del consumidor y su proceso de decisión de compra se ha convertido en la punta de iceberg de las empresas que quieren que sus clientes tengan la elección por sus productos o servicios; estos dos grandes temas brindarán a las empresas y sus equipos de marketing puedan realizar estrategias y acciones adecuadas; es por ello que inicialmente desarrollaremos los conceptos de comportamiento y proceso de decisión de compra, para luego analizarlo con una visión evolutiva, es decir, cómo ha ido cambiando y cómo es el nuevo camino del consumidor.

Ferrell y Hartline (2012) nos refiere que "la capacidad de determinar la información profunda acerca de los clientes es un fenómeno medianamente reciente en marketing. Por ejemplo, hace 50 años la tecnología y el knowhow del marketing eran menos sofisticados" ( $p$. 152). De esta manera los mercadólogos de ese tiempo no podían entender por completo las necesidades y los deseos de los clientes por ello la necesidad e importancia del estudio del consumidor y su proceso de decisión de compra.

\section{Comportamiento del Consumidor}

Es importante iniciar explicando lo que se entiende por consumidor. Según la definición de la Real Academia Española (RAE.), define el concepto de consumidor como "persona que adquiere productos de consumo o utiliza ciertos servicios". Desde el punto de vista de marketing, Kotler y Armstrong (2012) refiere que el concepto del consumidor como, aquel que usa o dispone del producto o servicio que tiene pensado adquirir para satisfacer sus necesidades.

Abordar el concepto de comportamiento del consumidor con autores contemporáneos nos brinda información de partida. Para Mollá (2006) menciona que el comportamiento del consumidor de puede entender "como el conjunto de actividades que realizan las personas cuando seleccionan, compran, evalúan y utilizan bienes y servicios, con el objeto de satisfacer sus necesidades, actividades en la que están implicados procesos mentales y emocionales, así como acciones físicas" (p. 18). Según Rivera et al. (2009) define al comportamiento del consumidor como "proceso de decisión y la actividad física que los individuos realizan cuando buscan, evalúan, adquieren y usan o consumen bienes, servicios o ideas para satisfacer sus necesidades" (p. 36).

De las definiciones anteriores, se puede rescatar que el estudio del comportamiento del consumidor busca identificar cómo reacciona el consumidor tomando en cuenta sus procesos mentales y emocionales y cómo las empresas cada día se enfocan en cautivar a este consumidor, para concluir su objetivo de finalizar la decisión de compra y concretar la venta, por 
ello, tienen que investigar al consumidor bajo cuestionamiento como: ¿qué compra?, ¿por qué compra?, ¿cómo lo compra?, ¿cuándo lo compra?, ¿dónde lo compra?, ¿cuánto compra?, ¿cómo lo utiliza?; todo este proceso no es sencillo, debido a que implica el análisis minucioso de su comportamiento, su decisión de compra y el proceso a seguir para la adquisición de un bien o servicio. Si la empresa analiza el comportamiento y proceso de decisión de compra logrará atraer a su cliente para luego retenerlo y fidelizarlo.

Por otro lado, cobra relevancia la revisión de algunas teorías o modelos de conducta del comportamiento del consumidor, así tenemos:

- Teoría económica de Marshall, considera que las decisiones de compras son el resultado de cálculos económicos, bien pensado, es decir, racionales y conscientes no tomando en cuenta los atributos del producto. Rivera et al. (2009) refiere que el consumidor comprará productos que le proporcionen utilidad de acuerdo a sus gustos, cuestionamientos simples como, a menor precio, mayor venta. En conclusión, el consumidor maximizará su consumo en función del costo-beneficio que obtenga.

- Teoría de conducta de aprendizaje de Pavlov, nos refiere Whaibe et al. (2013) que en el descubrimiento de los principios de aprendizaje de la relación estímulo y respuesta se basa en cuatro conceptos como: impulsos, claves, respuestas y reacciones. Por ello, el trabajo que realicen los mercadólogos de publicidad tiene que enfocarse en palabras, colores, imágenes claves que puedan finalmente estimular el impulso del consumidor.

- Teoría Psicoanalítica de Freud, según la teoría de Freud, nos dice que en cada persona existe una energía psíquica distribuida en las facetas de la personalidad que se proyecta hacia la satisfacción de necesidades y que no necesariamente se llevan por factores económicos. Para Schiffman y Kanuk (2005) la teoría de Freud se desarrolló sobre premisas de que las necesidades o impulsos es lo fundamental de la motivación y la personalidad humana, basándose en los recuerdos y experiencias en la primera infancia, el análisis de los sueños y naturaleza específica de los problemas. Finalmente, cobra importancia el grado de enfrentar las diferentes crisis que experimentó el individuo en su etapa inicial de vida.

- Teoría Psicológico Social de Veblen, según Martinez et al. (2013) nos menciona que el hombre es considerado como un animal social, adaptado a normas de su cultura, sus deseos y conducta están forjados con afiliaciones actuales o pertenecer a aquello que quiere alcanzar. Entre los factores que considera: cultura, grupo de referencia, familia, religión, política y relaciones humanas. Concluyendo que, el hombre está motivado por la búsqueda de prestigio.

- Teoría de la motivación de Maslow, es una de las teorías que más se usa en temas de marketing por la cual Maslow intentó buscar motivaciones y las ordenó en forma jerárquica o de urgencia. Así tenemos, que existen las necesidades fisiológicas o de supervivencia (hambre, sed), necesidades de seguridad o protección, necesidades sociales (ser querido y reconocido pertenecer a un grupo), necesidades de estima (autoestima, status, reconocimiento) y las necesidades de auto realización o desarrollo máximo (autodesarrollo y realización). Maslow concluye que mientras una persona no se encuentre satisfecha a una necesidad dentro de la pirámide no podrá avanzar al siguiente nivel, es decir, que las necesidades no satisfechas influyen en el comportamiento del consumidor.

De las teorías revisadas los mercadólogos las usan para preparar su plan de marketing con el uso de las 4Ps, desarrollando productos, fijando precios, revisando canales adecuados de distribución y lanzamiento de campañas publicitarias, adelantándose al comportamiento del consumidor; pero a la vez se necesitan de nuevas teorías y enfoques para seguir avanzando 
en este conocimiento y estar un paso delante de los cambios de este comportamiento.

Kotler y Armstrong (2012) nos refiere los factores que afecta al comportamiento del consumidor, "las compras del consumidor reciben una gran influencia de las características culturales, sociales, personales y psicológicas" (p. 135). Analicemos estas características o factores:

- Los factores culturales determinadas por las normas y costumbres, creencias aprendidas en sociedad y que finalmente llevan a comportamientos comunes. Los valores culturales constituyen guías para el comportamiento, cada sociedad tiene su cultura e influencia en su comportamiento de compra.

- Los factores sociales, afectan al comportamiento del consumidor ya que recibe la influencia de estos, como sus pequeños grupos, su familia, sus papeles sociales y su estatus, llamados también grupos de referencia, con los cuales el consumidor va a interactuar y se va a identificar, así tenemos el pertenecer a un partido político, equipo deportivo, algún colegio profesional y otros como la familia, grupo de amigos o grupos de aspiración a los que se desea pertenecer. El comportamiento de la familia también ha sufrido una evolución a través de los años con la participación activa de la mujer y la decisión de los jóvenes y niños en el núcleo familiar.

- Los factores personales, podemos mencionar que las decisiones de compra se ven afectadas por características personales como la edad, etapa del ciclo de la vida, ciclo de vida familiar, ocupación, situación económica, estilo de vida, personalidad, accesibilidad al internet entre otras.

- Los factores psicológicos, que influyen en las decisiones de compra: motivación, percepción, aprendizaje, creencias y actitudes. Finalmente podemos considerar que existe una relación entre el comportamiento del consumidor y la personalidad del individuo al momento de realizar o tomar una decisión de compra.
Con la revisión de los factores que afectan el comportamiento del consumidor Rivas y Esteban (2010), menciona que "si las culturas difieren y si los consumidores se comportan de modo diferente, las estrategias de marketing también deben adaptarse a los distintos entornos que se puedan identificar" (p. 166). De esta manera, se hace hincapié a lo dinámico que puede ser trabajar las estrategias de marketing en la actualidad.

\section{Comportamiento de Decisión de Compra}

Para comprender el concepto de comportamiento de decisión de compra Kotler y Armstrong (2012) nos mencionan "las decisiones más complejas suelen requerir de un número de participantes mayor en la compra y mayor análisis por parte del comprador" (p. 150). Por ello, el comportamiento en la decisión de compra se vuelve complejo y variará de acuerdo al producto o servicio que se esté adquiriendo; no es lo mismo adquirir un par de zapatillas que comprar un televisor con lo último en tecnología.

Revisemos la propuesta de Kotler y Armstrong (2012) en cuanto a los tipos de comportamiento en la decisión de compra basada en los grados de participación por parte del comprador y la magnitud de diferencia entre las marcas:

- Comportamiento de Compra Complejo, la participación del consumidor es mayor; pero se apoya en las diferencias significativas entre las marcas; este consumidor se involucra mucho más por ser productos de mayor valor, por ello está dispuesto a aprender o informarse más y tomar una decisión bien fundamentada. Un ejemplo a tener en cuenta es la adquisición de una laptop para un estudiante de diseño.

- Comportamiento de compra que reduce la disonancia, la participación también es mucho mayor; pero no logra encontrar diferencias significativas entre las marcas, refiere a compra de productos poco frecuentes y riesgosos. Así podemos considerar la compra de un equipo de aire acondicionado y todo su proceso, selección, compra, recepción del producto, instalación y servicio técnico cobrando importancia el servicio posventa. 
- Comportamiento de compra habitual, se presenta con una baja participación del consumidor y poca diferencia entre las marcas, el consumidor no busca información del producto, ni evalúa sus características; es una compra sencilla, habitual. Aquí aplica la compra de productos de consumo masivo que se realizan de forma habitual en un supermercado, el consumidor tomará en cuenta lo inmediato que encuentra en una góndola, cobrando importancia el layout del producto en punto de venta.

- Comportamiento de compra que busca variedad, se caracteriza por la escasa participación pero tiene diferencias percibidas entre las marcas; el consumidor no evalúa y finalmente puede cambiar fácilmente de marca, valorando la mayor variedad y es menos leal. Es importante destacar el desarrollo de la marca propia del establecimiento o cadena debido a que este cliente puede elegirla que a pesar de estar cerca de una marca líder. Así tenemos, la compra de galletas de soda, azúcar es indistinta en seleccionar una marca en especial.

\section{Proceso de Decisión del Consumidor}

Habiendo revisado el comportamiento del consumidor, su comportamiento de decisión de compra, factores que influyen y los afecta, pasamos a exponer cómo es el proceso de decisión del consumidor, como ha ido evolucionando en el corto plazo y cómo las empresas pueden aprovechar mejor este conocimiento.

Para Rodriguez y Rabadán (2013) nos menciona que "los consumidores, en general, reconocen la necesidad, ya sea por motivos personales o por influencias del entorno" (p. 330). Por ello hay que analizar no sólo variables internas y externas del consumidor en el proceso de decisión de compra.

Kotler y Armstrong (2012) nos refieren que "el proceso de decisión de compra se inicia mucho antes de la compra real, y que continúa mucho tiempo después" (p. 152). Por eso, cobra mucha importancia el estudio del proceso de decisión de compra en la preventa, durante y su posventa, para ello Kotler propuso las cinco etapas del proceso la cual repasaremos:

- Reconocimiento de las necesidades, el consumidor reconoce su necesidad pudiendo ser de origen de estímulo interno o externo. Por ello es importante conocer a su consumidor realizando investigaciones, reconocer cuáles son sus dolores o carencias y llevarlo a la siguiente etapa de búsqueda de la información y considere a la empresa como primera alternativa de consulta.

- Búsqueda de información, el consumidor busca activamente información del producto o servicio que lo satisfaga, la información es obtenida de muchas fuentes como personales (familia, amigos, vecinos), fuentes comerciales (publicidad, vendedores entre otras), fuentes públicas (búsquedas en medios de comunicación y en internet) y las fuentes empíricas (manipulando, examinando el producto). Conforme tenga mayor información, será una compra más consciente y con conocimiento del producto.

- Evaluación de alternativas, en esta etapa el consumidor diferencia las alternativas encontradas, es una etapa compleja porque tiene que procesar toda la información recibida y elegir una marca, producto o servicio determinado.

- Decisión de compra, el consumidor ya adquiere la marca preferida o elegida luego de toda la evaluación realizada. Es aquí donde influye las actitudes de su entorno, es decir, las personas, cuyos comentarios pueden ser positivas o negativas de manera que fortalecerá su decisión de compra o cambio de la marca.

- Comportamiento posterior a la compra, una vez concretada la compra el consumidor determinará si está satisfecho o no, se busca que la brecha entre la expectativa y el desempeño sea lo mínimo. Es una etapa que cobra relevancia debido a que el consumidor generará referencia de la marca por su experiencia de compra. 
Los consumidores de la actualidad están más empoderados pueden encontrar los productos que quieran, a los precios que mejor les convenga, con despachos en diferentes puntos donde esté más cerca o conveniente, pueden realizar su compra a nivel mundial y lo pueden hacer 24/7; la omnicanalidad juega un papel importante. Los consumidores cuentan con un acceso de información increíble donde pueden encontrar evaluaciones o comentarios de otros consumidores que compraron antes el producto.

Por otro lado, tenemos a los comerciantes, empresas o negocios que frente a estos hechos han tenido que cambiar su forma de vender, ampliando el surtido de productos, personalizando sus servicios, despachos, tiempos de entrega que logre una experiencia muy especial y pueda continuar por la etapa de la recomendación o lealtad de su marca. Esteban Millat (2011) refiere que las plataformas de navegación se debe facilitar experiencias al consumidor, proponiendo formas entretenidas y agradables, hacer sentir al consumidor la percepción de tener en control en el entorno. Los consumidores responden mucho más rápido a la comunicación digital que tradicional. Bajo este contexto, Kotler et al. (2017) presenta cómo comprender el proceso de compra del consumidor de hoy, si bien el modelo AIDA: atención, interés, deseo y acción; es un clásico del sigo XIX cuyo pionero de la publicidad y ventas es E.St. Elmo Lewis nos servirá como base al análisis de hoy pero ahora con un modelo evolucionado cuyo aporte lo da Derek Rucker de la Kellog School of Management quien hace una modificación de las 4 As llamándolas: Consciente, actitud, acto y acción, agregando la recomendación. Así es como plantea, Kotler et al. (2017) "Los clientes aprenden sobre una marca (consciente), les gusta o no les gusta (actitud), deciden si la compran (actúan) y finalmente deciden si vale la pena repetir la compra (actuar de nuevo)" (p. 46), todo este proceso que menciona, es conocido como el embudo o funnel de venta por el cual atraviesa el consumidor en su nuevo camino de decisión de compra.

Toda esta conectividad en la que vivimos lleva a un cambio o actualización en cuanto a las $5 \mathrm{As}$, así tenemos que el proceso de decisión de compra del consumidor de hoy sigue los siguientes pasos:
- Conocimiento/Acceso a la marca (Aware), es el punto inicial en donde el consumidor está expuesto pasivamente a una serie de marcas a través de la publicidad, redes sociales, familia y sus experiencias vividas. En este punto tenemos que revisar cómo el consumidor se entera o conoce el producto, si estamos generando el contenido necesario para que pueda ser la primera alternativa a tener en cuenta.

- Atraer (Appeal), aquí el consumidor procesa la información publicitaria y desarrolla una preselección, considerando opiniones de su entorno más cercano como son sus amistades, redes sociales, comunidades, blogs entre otros. Aquí ya se despierta la atracción, curiosidad e interés hacia una marca determinada

- Asesoramiento / Preguntar Pedir (Ask), el consumidor buscará más información sobre la marca que le interesa, también consultará a sus círculos más cercanos y es en este momento que la marca producto o servicio debe estar más expuesta en canales o medios digitales.

- Acción (Act), una vez ya informados, asesorados los consumidores pasan a la acción; no sólo se limitan a la compra sino al uso e interacción con la marca y comentar en redes sociales sobre lo adquirido.

- Recomendar / Apoyo (Advocate), es aquel momento en el cual el consumidor está dispuesto a recomendar la marca. Esta etapa cobra importancia la participación de las redes sociales, por las acciones de marketing que esté realizando la empresa, es lo que se conoce como los "reviews" o comentarios positivos o negativos que hace el consumidor acerca de la marca o producto; quién no ha consultado una opinión antes de comprar.

En resumen, se ha elaborado un cuadro matriz ver Figura 1, que muestra los procesos de decisión de compra desde el punto de vista 


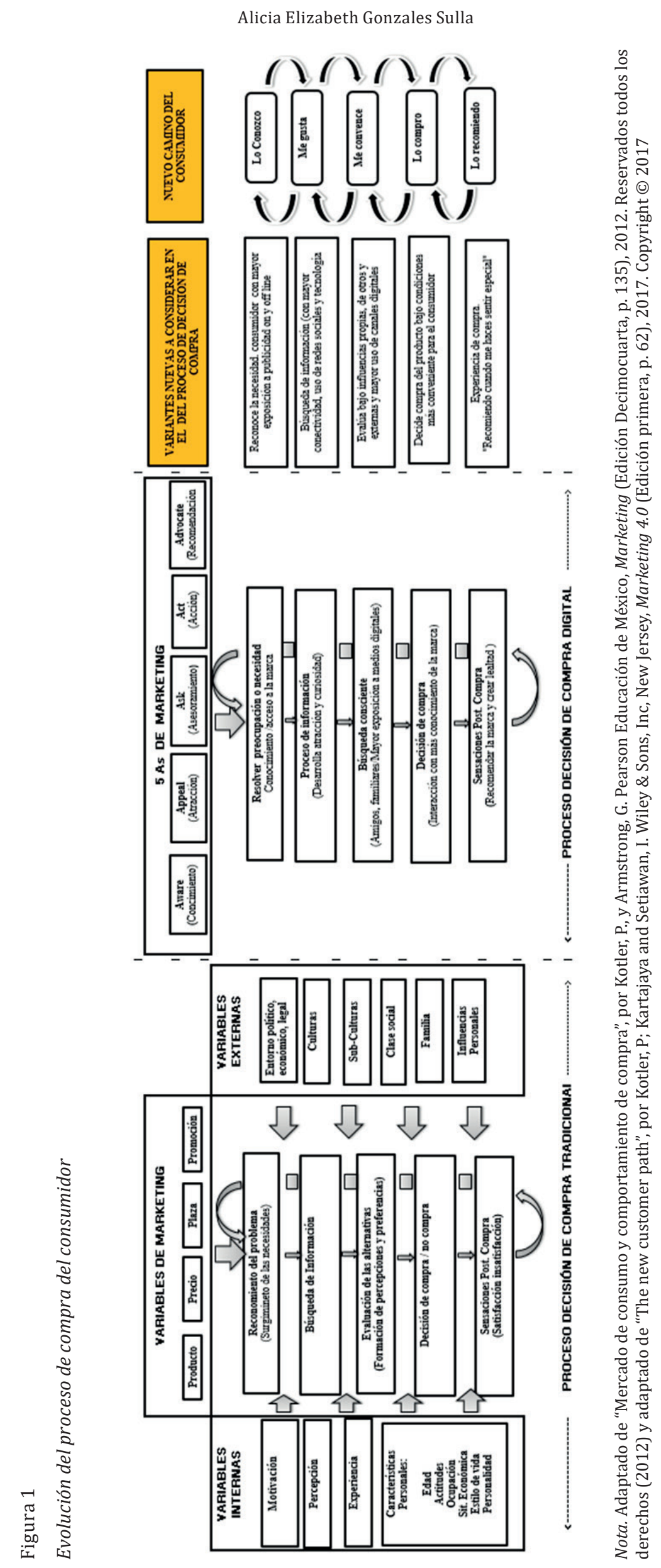


de marketing tradicional y digital. Según Kotler et al. (2017), el objetivo es rastrear el comportamiento del consumidor posterior a la compra y medir la retención del consumidor y llegar a la lealtad con los clientes.

Entre las diferencias que podemos encontrar en ambos proceso tenemos que el reconocimiento de la necesidad ahora se encuentra más expuesta a publicidad permanente que ni el mismo consumidor se da cuenta (mayor exposición publicitaria on y off line); la búsqueda de información ahora se realiza con mayor interconexión haciendo uso de medios digitales como son las redes sociales y los dispositivos tecnológicos considerando opiniones de su entorno más cercano; la evaluación de alternativas ahora pasa por tres tipos de influencias según lo describe Kotler et al. (2017), influencias personales donde están las experiencias propias, juicios personales e interacción con la marca, influencia de otros donde se encuentra la familia, amigos y redes sociales, y la influencia externa donde se encuentra la publicidad. La decisión de compra del consumidor de hoy le permite gozar de una serie alternativas de la cadena de valor que tendrá en cuenta para este paso, hoy en día el tema logístico cobra un papel decisivo en la experiencia del consumidor; finalmente la satisfacción post venta se traduce en experiencia del consumidor que su participación en todo este proceso sea la mejor para que pueda crear lealtad a la marca y poder recomendarla. Toda este nuevo o camino del consumidor se da bajo un ciclo de retroalimentación permanente que hay que mantenerlo activo.

\section{El nuevo camino del consumidor}

Conocer a profundidad el nuevo camino del consumidor de hoy es el reto que tienen las empresas, saber dónde se encuentra, qué le interesa y finalmente qué lo motiva a concretar la compra es un tema valorado, el cual requiere de mucho análisis en la información. Como se revisó en el punto anterior, el proceso de decisión de compra, se está enfrentando a cambios significativos en varios frentes cómo son la forma de comprar sus productos, la búsqueda de información, la revisión de alternativas de compras a considerar, la forma de recibir sus productos, adicionando a ello el uso de canales digitales para ello las empresas están aplicando estrategias omnicanal como alternativa para estar presente en todos los puntos de contacto y que su experiencia de compra sea la mejor.

Desde la posición de Mercado et al. (2019) en su artículo de comportamiento del consumidor on line con el que concluye "el análisis del comportamiento del consumidor puede ayudar a la creación de estrategias para la toma de decisiones efectivas mediante su aplicación en el diseño de plataformas de compra en línea" (p. 14). Por ello, es necesario conocer herramientas actualizadas para el análisis del consumidor, así tenemos:

- Buyer Persona, consiste en la elaboración de una descripción abstracta de un "consumidor ideal", con variables demográficas, motivaciones, preocupaciones, historias personales, retos entre otras), el contar con esta información servirá de base para la estratégica de marketing y venta.

- Mapa de Empatía (Emphaty Map), esta herramienta nos ayuda a comprender su interior del consumidor, sus puntos de vista; analiza seis aspectos de los sentimientos del consumidor, qué piensa y siente (sus dolores o necesidades), qué oye (que dicen acerca de lo que va a decidir), qué ve (en su entorno, amigos y mercado) y qué dice y hace (su actitud en público, su comportamiento hacia los demás).

- Viaje del consumidor (Customer Journey), no es un recorrido físico sino conceptual, en el cual no necesariamente el consumidor seguirá el paso a paso ya que dependerá del rubro o categoría de producto que se esté decidiendo comprar. En este viaje, se cuenta con detalles de la experiencia del consumidor desde el momento que tiene la necesidad hasta después de adquirir el producto. El estudio de este recorrido cada vez es más importante porque le da la oportunidad de mejorar procesos en este viaje del consumidor y así ofrecer más valor y satisfacción al consumidor.

- Mapas de calor (Heatmaps), permite identificar los puntos que tienen mayor 
atracción para el consumidor, es toda la ruta que ha seguido al momento de iniciar su proceso de compra.

Las herramientas mencionadas tienen que ser analizadas cuidadosamente y a mayor profundidad, en el presente artículo sólo se ha tocado los puntos en forma general y sirven de partida para otros estudios de investigación en el desarrollo del consumidor objetivo; los puntos de contacto por el cual recorre el consumidor y su experiencia en cada uno debe llevarnos a conocerlo más; solo las empresas que dominen las herramientas podrán generar nuevas acciones en el momento indicado y mejores experiencias para el consumidor y así competir mucho mejor.

Para concluir, Roberts (2005) nos recuerda como es este nuevo consumidor "el nuevo consumidor está mejor informado, es más crítico, menos leal y más difícil de entender. Se ha fundido en una nueva población multigeneracional, multiétnica y multinacional" (p. 35). Por lo tanto, el desafío actual es trabajar con este consumidor muy de cerca y lograr cautivarlo creando vínculos del consumidor con la marca, producto o servicio.

\section{CONCLUSIONES}

Estamos frente a una realidad innegable, el mundo se ha digitalizado a una velocidad exponencial y las empresas están dando paso a cambios importantes como trabajar un mundo físico y virtual o ambos, todo ello indica reformular nuestros conceptos, estrategias, propuesta de valor y relaciones con los clientes.

Los consumidores en su proceso de decisión de compra atraviesan diferentes etapas progresivas y secuenciales, algunas veces serán más breves y otras podrán saltarse debido al tipo de producto o servicio que estén adquiriendo, es por ello que analizar cada una de estas etapas tiene un valor significativo, para conseguir guiar al consumidor en este camino y así lograr, ser la primera opción a tomar en cuenta ya no se trata de una simple venta, este consumidor debe llegar a recomendar la marca o producto.

La aplicación de estrategias de marketing no solo debe quedarse en estrategias de segmentación, posicionamiento y diferenciación sino sumarle las 5 As actualizadas, con la cual se consigue conocer el camino que sigue el consumidor de hoy, aplicadas tanto al mundo físico como virtual, adicionando a ello las estrategias de omnicanalidad cuyo papel cobra importancia en este camino, facilitando los puntos de encuentro del consumidor con la empresa en la adquisición de los productos o servicios.

La experiencia de compra del consumidor es vital en este nuevo camino del consumidor, se debe superar las expectativas que tiene el cliente frente a la compra que está realizando y lograr cautivarlos y emocionarlos con un servicio confiable, personalizado, encontrando siempre soluciones, estas experiencias que tiene el consumidor siempre serán recordadas y apreciadas siendo elemento de lealtad y compromiso con la marca.

Delimitar los conceptos de comportamiento del consumidor, comportamiento de compra y proceso de decisión compra del consumidor, plantea encontrar las diferencias significativas en este nuevo camino del consumidor; las empresas que logren hacerlo podrán gozar de mayor rentabilidad y dirigir sus esfuerzos en conocer más a su consumidor, adaptarse a él y acompañarlo, no sólo para obtener un resultado de ventas sino crear una relación o vínculo de cercanía con el consumidor.

Finalmente, viviendo una coyuntura como la del COVID-19 la evaluación del comportamiento del consumidor va dirigido al uso de canales y medios digitales y tanto las grandes, medianas o pequeñas empresas tienen que estar en la ola de participar de estos modelos digitales para la venta de sus productos haciendo buen uso de la analítica de datos que nos brindan los propios consumidores en este nuevo camino.

\section{REFERENCIAS BIBLIOGRÁFICAS}

Esteban Millat, I. (2011). Flujo y comportamiento del consumidor en línea: Un análisis empírico de las experiencias de consumos de productos formativos. Barcelona, España: Universitat Oberta de Catalunya.

Ferrell, O. C., y Hartline, M. D. (2012). Estrategia de Marketing (Vol. 5ta. Edición). México: Cengage Learning Editores S.A.

Kotler, P., y Armstrong, G. (2012). Marketing. México: Pearson Educación de México. 
Kotler, P., Kartajaya, H., y Setiawan, I. (2017). Marketing 4.0. New Jersey: Wiley \& Sons, Inc.

Martinez, C., Ruiz, J., M, L., y Verján, R. (2015). El comportamiento del consumidor en internet bajo el modelo psicológico social de Veblen. Revista global de negocios, Vol.3 No.5, 101-112.

Mercado, K., Perez, C., Castro, L., y Macias, A. (2019). Estudios cualitativos sobre comportamiento del consumidor en las compras on line. Información tecnológica.

Mollá, D., Berenguer, C., Gomez, B., y Quintanilla, P. (2006). Comportamiento del Consumidor. España: UOC.

Real Academia Española. (Sin fecha). Diccionario de la lengua española. https://dle.rae.es/consumidor? $m=$ form

Rivas, A., y Esteban, G., (2010). Comportamiento del consumidor. Decisiones y estrategias de marketing (Vol. 6ta Edición). Madrid: ESIC Editorial.
Rivera, C., Arellano, C., y Molero, A. (2009). Conducta del consumidor: Estrategias y políticas aplicadas al marketing (2da ed.). Madrid - España: ESIC Editorial.

Roberts, K. (2005). Time changes everything. En lovemarks: el futuro más allá de las marcas. Barcelona: Urano.

Rodriguez, D., y Rabadán, B. (2013). Proceso de decisión del consumidor. Factores explicativos del visionado de película en sala de cine de los jóvenes universitarios españoles. Barcelona, España: Universitat Internacional de Catalunya.

Shiffman, L. G., y Kanuk, L. L. (2005). Comportamiento del consumidor (Vol. 8va Edición). México: PEARSON EDUCACIÓN.

Whaibe, E., Garcia, P., y Castillo, R. (2013). Teorías del comportamiento del consumidor. Apizaco, México: Universidad pública de Apizaco 